\title{
Trabajo docente y narrativa: Las dimensiones del Ser docente
}

\author{
Teachers' work and narrative: the dimensions of the being
}

Trabalho docente e narrativa: as dimensões do Ser docente

María PAUla CARRERAS (Da

PAULA BeLló (iDb

CYNTHIA MARÍA TORRES STOCKL (iDc

\section{Resumen}

El Ser docente se define en torno a cuatro grandes dimensiones que funcionan entre sí como capas concéntricas y cada una de ella tiene una dinámica distinta. El Ser Ontológico, el Ser Ético, el Ser Pragmático y el Ser Político se conjugan de tal manera que permiten conjuntamente delimitar la esencia del fenómeno del trabajo docente. A estas conclusiones se ha arribado en un estudio fenomenológico que tuvo por objetivo relevar la experiencia de docentes latinoamericanos en torno a su quehacer, a través de la escritura de experiencias narradas a colegas en cartas. Con esta finalidad, se contó con la participación voluntaria de 15 docentes, 10 procedentes del Norte de Argentina y 5 del sur de México seleccionados intencionalmente. Dichos docentes fueron invitados a escribir una carta dirigida a un colega extranjero narrando acerca de la experiencia de su trabajo. Este corpus obtenido se analizó mediante el método Colaizzi (1978) obteniendo un total de 245 declaraciones significativas extraídas luego de las primeras lecturas, 239 significados formulados, 56 agrupamientos temáticos y, 17 temas emergentes que devienen en las 4 categorías teóricas que describen la esencia del fenómeno abordado.

a Universidad Nacional de Tucumán, San Miguel de Tucumán, Tucumán, Argentina. Doctora en Sociología y Ciencias Políticas, e-mail: paulacarreras@hotmail.com

bUniversidad de Talca, Linares, Chile. Doctora en Educación, e-mail: paula.bello@utalca.cl

c Universidad de Talca, Linares, Chile. Dra. (c) en Humanidades, e-mail: cynthiatorresstockl@hotmail.com 
Palabras clave: Trabajo docente. Narrativa. Dimensiones del Ser docente.

\begin{abstract}
Teachers' Internal Being is defined according to four general dimensions that interact among them like concentric layers, each having a different dynamic. The Ontological Being, the Ethical Being, the Pragmatic Being, and the Political Being, combine among each other and help establish limits to the essence of the teachers' work phenomenon. Such are the conclusions generated by a phenomenological study, in which the main objective was the description, analysis and interpretation of Latin-American teachers' experiences and reflections about their daily lives and work. A total of 15 teachers, ten from Northern Argentina and five from Southern Mexico, wrote letters and exchanged them. This corpus was analyzed by means of the Colaizzi Methods (1978). The analysis resulted in 245 significant statements extracted from the first readings, 239 formulated meanings, 56 thematic clusters and 17 emergent themes that become into the four theoretical categories that describe the essence of the phenomenon.
\end{abstract}

Keyword: Teachers' Work. Narratives. Dimensions of the Being (teachers).

\title{
Resumo
}

O ser docente define-se entorno de quarto grandes dimensoes que funcionan entre sí como como camadas concentricas e cada uma de las tem uma dinamica distinta. O ser ontológico, o ser ético, o ser pragmático e o ser politico conjugados de tal forma que permitem delimitar a essencia do fenómeno do trabalho docente. As conclusoes chegou-se um estudo fenomenológico que teve por objetivo relevar a experiencia de professores latinoamericanos em torno de sua actividade, a través da escrita de experiencias narradas a colegas em cartas. Com esta finalidade, contou-se com a participacao voluntária de 15 docentes, 10 provenientes do Norte da Argentina e 5 do sul do México, selecionados intencionalmente. Esses professores foram convidados a escrever uma carta dirigida a um colega estrangeiro narrando sobre a experiencia de seu trabalho. Este corpus obtido foi analisado pelo método Colaizzi (1978) obtendo um total de 245 declaracoes significativas extraídas apos as primeiras leituras, 239 significados formulados, 56 agrupamentos temáticos e, 17 temas emergentes que se tornam em todas as 4 categorias teóricas que describen la esencia del fenómeno abordado.

Palavras-chave: Trabalho docente. Narrativa. Dimensões do Ser docente.

\section{Introducción}

La profesión docente es producto de sujetos concretos ubicados en un tiempo y espacio definidos. La misma supone un hacer complejo que involucra la articulación de un 
conjunto de saberes que incluyen, saberes de la experiencia, saberes pedagógicos e ideológicos, y saberes curriculares disciplinares, que determinan su práctica efectiva. Como sujeto y actor educativo, el docente es poseedor de esos saberes, creencias, e imaginarios orientadores de sus prácticas que le otorgan sentido y significado a su hacer cotidiano (AGUAYO-ROUSSELL, 2020). Los docentes comparten saberes comunes acerca de su profesión, independientemente del nivel educativo o el lugar donde se ubiquen las instituciones.

La comprensión del conocimiento, valores, y actitudes que constituyen la especificidad del trabajo docente, así como los procesos involucrados en la construcción social de su ser y de su profesión en un espacio social determinado, proveen de sentido y orientación a su práctica en términos de saberes propios de la docencia. Achilli (2008) define a la práctica docente como un conjunto de actividades, interacciones, y relaciones que configuran el campo laboral del sujeto docente en condiciones institucionales y sociohistóricas determinadas. Asimismo, Barco (1996) afirma que la práctica docente adquiere una significación específica, tanto para la sociedad, como para quien la ejerce. La docencia, si bien está definida según su significación social y particular por la práctica pedagógica, va mucho más allá de ella al involucrar una compleja red de actividades y relaciones que la traspasan, las que no pueden dejar de considerarse si se intenta conocer la realidad vivida por el docente. De esta manera deviene fundamental identificar cuestiones relativas al contexto externo, a la vez, que aquellas vivenciadas desde perspectivas personales lo que permite entender la trama de percepciones de los actores sociales que conforma el ser y el hacer docente en el contexto socio histórico determinado (SAUCEDO; ALCALÁ; FERREYRA, 2019).

Edelstein (2000) sostiene que cuando se hace referencia a la acción docente, se la contextualiza generalmente en el aula. Esta última parece como un microcosmos del hacer, un espacio privilegiado donde se despliega la acción. Se trata un lugar casi único, donde la acción docente se encuentra constreñida, y sobre el cual existe desconocimiento de las acciones que suceden en su interior. Allí, por ejemplo, se articulan múltiples determinaciones de orden extra áulico que se inscriben en la estructura y dinámica institucional y que amarran a los actores, aunque sean individualizables, a una historia social compartida. 


\section{El Mal-Estar Docente: La multiplicidad de actividades y el desgaste docente}

Los profesores se enfrentan a la paradoja del reconocimiento de su rol que es clave en los procesos de enseñanza-aprendizaje y perciben una creciente desvalorización de su quehacer cotidiano. Esto implica una permanente tensión identitaria. Estudios realizados con docentes indican, que estos se auto-perciben como sujetos que realizan una profesión que recibe bajas remuneraciones, que realiza un trabajo agobiante y que no cuenta con el tiempo necesario para actividades esenciales como: la planificación, preparación de materiales, evaluación, superación profesional, organización de trabajo en equipo, atención de estudiantes y sus familias, entre otras actividades, además de acciones adicionales a la enseñanza en el aula (BELLEI; VALENZUELA, 2010; BELLÓ; TORRES STÖCKL; CARRERAS, 2019). Investigaciones actuales indican que el docente está envuelto en una realidad que le demanda respuestas rápidas y efectivas, cambios y mejoras, así como el desempeño de multifunciones -enseñar, contener, planificar, coordinar, mediar conflictos$y$, al fin, la inmersión en un estilo de vida que suele volverse vertiginoso. Asimismo, surgen nuevas situaciones de riesgo para los docentes -circunstancias producidas por la cultura contemporánea-, entre las que se pueden incluir la sobrecarga laboral, el síndrome de 'burnout' docente, además de requerimientos tales como la profesionalización, capacitación y perfeccionamiento que constituyen parte de las exigencias más importantes a las que los docentes deben enfrentarse en la actualidad (AGUIRRE; APARICIO; MARSOLLIER, 2010; CARRERAS et al. 2017).

Frente a esta situación problemática, las políticas docentes no ocupan el centro efectivo de las prioridades debido a que se consideran de alto costo cuando se propone beneficiar al conjunto de la docencia. Corrales (1999) y Navarro (2006) consideran que estas políticas se encuentran en el polo de las políticas difíciles. Las mismas no se han centrado en la acción del docente específicamente, como así tampoco han considerado la articulación que exige la práctica docente con las condiciones del contexto, dejando de lado la subjetividad 
del docente que ejerce la docencia. Es decir, las políticas no consideran la importancia de las condiciones socioculturales en el desempeño docente, así como no tienen en cuenta la dinámica de la subjetividad del profesor en la acción educativa (SOUSA; VILLAS BÔAS, 2011a). Por lo tanto, en algunos países de Latinoamérica como Argentina, Ecuador, Brasil, Cuba y México el trabajo docente se revela como un problema a investigar.

\section{La Búsqueda de Nuevas Perspectivas de Análisis}

En relación con lo descrito anteriormente, las perspectivas descontextualizadas que han sido utilizadas para evaluar el trabajo docente, sin tener en cuenta el análisis de la subjetividad del profesor, proporcionan una evaluación parcial de sus acciones y crean altas expectativas en relación con su actuación. Como resultado, el docente desarrolla un sentimiento de culpabilidad y frustración que no conduce a un compromiso con la docencia responsable (SOUSA; VILLAS BÔAS, 2011a; SOUSA; VILLAS BÔAS, NOVAES, 2011b). Los docentes configuran su manera de ser y asumirse como tales desde la presencia de discursos instituidos que se encarnan en la subjetividad. Por lo tanto, la subjetividad docente se construye en función de un discurso estructurante e instituyente. Esto explica que la constitución de la subjetividad del docente se basa en la función social que se le asigna a la tarea educativa en cada momento histórico (PRIETO QUEZADA; CARILLO NAVARRO, 2018).

Esto significa que es importante develar cómo comprenden y experimentan los docentes el sentido de su tarea, los factores que conducen a su buen desempeño, los vínculos que mantienen con su profesión y que definen su identidad social, el saber que los constituye como docentes, y las expectativas que tienen en relación con su futuro profesional, así como también las que orientaron su elección profesional. Con base en la comprensión sobre cómo piensan los docentes, es necesario deconstruir y construir las relaciones que se establecen entre los saberes científicos y los saberes del sentido común, en vinculación con el área educativa. 


\section{La Educación Docente: Un Camino Hacia el Desarrollo de la Subjetividad}

Es necesario entender el carácter, contextualizado y situado de la labor docente. En este respecto, Sousa y Villas Boas (2011a) se refieren a la necesidad de que, las políticas de educación profesional de docentes consideren la interacción existente entre las experiencias de vida de los futuros docentes y las actividades curriculares que serán desarrolladas en un contexto específico. Simultáneamente a la necesidad y la urgencia de recibir una buena educación, con el objetivo de conseguir buenas condiciones de trabajo y salario digno, es también necesario pensar en la preparación que el futuro docente necesita para comprender la subjetividad que se construye en las escuelas, tanto en relación con los alumnos, como con los demás colegas docentes. El objetivo último sería desarrollar un posicionamiento político crítico, afín a la construcción de políticas de subjetividad que consideren a los agentes sociales implicados en ellas a fin de asegurar su efectividad. En resumen, sería esperable que la educación profesional de docentes contemple el problema de lo cotidiano, del contexto, de las creencias y de las emociones y ofrezca estrategias de cómo encararlas (TEDESCO, 2004).

La subjetividad docente es un proceso abierto a las interacciones constantes de la vida cotidiana en la que están implícitos los elementos institucionales, políticos, sociales y culturales en los que vivimos. La misma se genera entre procesos colectivos e individuales, es una construcción, y de ello se desprende que los individuos son constructos, artificios, invenciones que se valen de máscaras para exponer lo que cada uno cree ser y quiere ser. La subjetividad es también un constructo-construyéndose continuamente, la subjetividad es abierta, no definida por completo y no determinada de antemano (PRIETO QUEZADA; CARILLO NAVARRO, 2018).

En el marco del Proyecto de Investigación de la Universidad Nacional de Tucumán Argentina -: "Bienestar - Malestar en torno al trabajo docente. Aporte para la construcción y validación de un instrumento destinado a su medición”, cuyo objetivo es contribuir teórica y metodológicamente a la interpretación del bienestar/malestar en torno al trabajo docente, y caracterizar los procesos de construcción y dinámicas de las subjetividades reconociendo las 
dimensiones relativas desde el propia referencia de los actores sociales, se incluyó la propuesta del subproyecto basado en la elaboración de narrativas y cartas por parte de docentes de diversos países Latinoamericanos, llamado "El quehacer docente: Intercambio de narrativas entre docentes latinoamericanos". El mismo fue propuesto, de manera de ahondar en el interés por el estudio del trabajo docente, ya expuesto en el desarrollo de la investigación bibliográfica, especialmente desde una mirada regional (norte argentino), nacional (Argentina), e internacional (Brasil y México). Asimismo, se planteó la necesidad de relevar los aspectos facilitadores y obstaculizadores del trabajo docente, así como, ahondar en la identificación de las representaciones sociales (pensamiento común o colectivo) en torno al trabajo docente.

Por lo tanto, el propósito fundamental de dicho estudio se centra en la exploración de las experiencias del quehacer docente, de manera de describir, analizar e interpretar el núcleo del fenómeno de la experiencia docente, contribuyendo a la discusión en torno a la construcción de la subjetividad social. La narrativa, género discursivo en el que se expresan relatos existenciales y, por tanto, que denotan una experiencia vivida por el sujeto en unas circunstancias espacio-temporales determinadas, nos permite recuperar aquellos relatos y narraciones que constituyen a los actores de la escuela como sujetos enseñantes. Los relatos evocan, provocan y convocan alrededor de su dignidad porque, en base a los dichos de un sujeto en particular, hablan de la valía de la condición humana en general (MEZA RUEDA, 2009). La narración de la propia experiencia permite vislumbrar las decisiones, saberes y supuestos que el docente toma en el proceso de su quehacer profesional; favorece la reflexión y la deliberación sobre dichas decisiones, saberes y supuestos y posibilita la socialización y la significación colectiva de lo narrado.

\section{Narrativa}

Las narrativas de los docentes incluyen los elementos característicos de la narrativa. La definición de la misma según Hinchman y Hinchman (1997, p. xvi) es usada cuando se trabaja con historias contadas por docentes: 
Las narrativas o historias en las ciencias humanas, pueden ser definidas provisoriamente como discursos con un orden secuencial claro que conecta eventos de manera significativa para una audiencia definida y que por lo tanto, ofrece una mirada profunda sobre el mundo y la experiencia humana del mismo.

Por lo que incluye tres elementos fundamentales presentes en las narrativas docentes: la temporalidad, la significatividad y el encuentro social. Goodson y Gill (2011) aseguran que existe una conexión entre la vida, de acuerdo a como es vivida, y la vida como es contada en las narrativas personales. Estos autores explican la presencia de la secuenciación de eventos y la significación personal de las experiencias al narrarlas y agregan la importancia del encuentro social, ya que todas las narrativas son contadas para una audiencia determinada y estan formuladas por la relación que existe entre el que cuenta y el que lee o escucha.

\section{Método}

\section{Tipo de estudio}

Este estudio cualitativo se enmarca dentro del enfoque metodológico de la fenomenología hermenéutica, que busca describir el significado común que diferentes individuos tienen de sus experiencias con un concepto o un fenómeno (CRESWELL, 2013; VAN MANEN, 1990). Heidegger (1996) describió a la fenomenología hermenéutica como una metodología filosófica que trata de descubrir el significado del "ser" y de la "existencia humana" por medio del análisis de la historicidad dese de la existencia. Para esto, reduce las experiencias individuales con un fenómeno a una descripción de la esencia universal del fenómeno`(VAN MANEN, 1990; MOUSTAKAS, 1994) y propone un análisis de datos que desarrolla procedimientos sistemáticos que se desarrollan desde unidades de significado mínimos y temas emergentes hasta la descripción detallada refiriéndose a “qué” y “cómo’ se experimenta el fenómeno (CRESWELL, 2013). Como conclusión de busca describir, analizar e interpretar la "esencia", el elemento culminante, o núcleo de un fenómeno (CRESWELL, 2013). 
Participantes

La muestra intencional no probabilística de participantes en este estudio está compuesta por 15 docentes, 10 procedentes del Norte de Argentina y 5 del Sur de México, de entre 23 y 63 años. El 93\% ( $\mathrm{n}=14)$ son mujeres mientras que el 7\%(n=1) son hombres. La totalidad de los participantes ejercen su tarea docente en el nivel primario, el $73 \%$ ( $n=11)$ en instituciones públicas y el $27 \%(n=5)$ en gestión privada.

Materiales

En los inicios del subproyecto basado en la elaboración de narrativas y cartas por parte de docentes de diversos países latinoamericanos, llamado "El quehacer docente: Intercambio de narrativas entre docentes latinoamericanos", se invitó a docentes de escuelas de nivel primario a participar. Por la intersección de los directivos de los centros educativos, se llevó a cabo la presentación del proyecto, incluyendo los objetivos de la investigación, el consentimiento informado, los datos sociodemográficos y una invitación a elaborar narrativas - cartas acerca de la experiencia de su trabajo docente, con el objetivo de intercambiarlas con colegas de otros países latinoamericanos.

\section{Procedimiento y análisis de datos}

Los docentes confirmaron su participación voluntaria mediante la firma del consentimiento informado, que asegura la confidencialidad de los datos recabados a lo largo del proyecto, y comenzaron con la escritura de sus cartas entre los meses de octubre y diciembre de 2018. La escritura de dichas cartas se llevó a cabo en sesiones presenciales en las escuelas, escenarios que ofrecen ambientes de confianza y comodidad a los docentes, por ser sus lugares de trabajo diario. Dichas cartas fueron retiradas de los centros escolares por los asistentes de investigación, previo acuerdo de día y horario.

Los datos sociodemográficos recabados se analizaron estadísticamente con el paquete SPSS versión 24. Por otro lado, para el análisis de las narrativas, se siguieron los pasos propuestos por el Método Colaizzi (SANDERS, 2003). En el primer paso se buscó obtener el sentido de cada narrativa. Para ello, se transcribieron la totalidad de las cartas, las que fueron leídas con detenimiento en tres oportunidades y sobre las que se fueron agregando 
reflexiones para facilitar el proceso de análisis temático. Seguidamente, se extrajeron las 'declaraciones significativas' que conforman el significado de la experiencia del trabajo docente. Estas declaraciones fueron transcriptas en forma textual, respetando la narrativa original del docente y permitieron la identificación de las vivencias vinculadas con la tarea docente. Asimismo, las 'declaraciones significativas' fueron numeradas para facilitar su posterior análisis. A continuación, se elaboraron los 'significados formulados', que consisten en declaraciones más generales o significados emanados de las declaraciones significativas extraídas de las transcripciones (COLAIZZI, 1978; SANDERS, 2003). El siguiente paso fue organizar los 'significados formulados' en 'agrupamientos temáticos', los que fueron fusionados en temas emergentes, que dieron lugar al nacimiento de categorías teóricas, sobre cuya formulación se discutió el proceso interpretativo.

Siguiendo el modelo de análisis planteado por Sanders (2003), con base en Colaizzi (1978), las transcripciones y análisis manuales facilitaron la constante inmersión de las investigadoras con los datos. La riqueza de dichos datos merece una larga inmersión en ellos, de manera que la descripción de la experiencia, refleje los pensamientos y sentimientos expresados por los docentes. Así mismo, las reflexiones emitidas por las investigadoras fueron incorporadas en el diario de reflexiones que se usó como insumo para el análisis y para explicar cómo se tomaron algunas decisiones interpretativas (SANDERS, 2003).

\section{Resultados}

El corpus textual obtenido consta de 15 cartas, 245 declaraciones significativas extraídas luego de las primeras lecturas, 239 significados formulados en base a las declaraciones significativas, 56 agrupamientos temáticos, denominados clusters en la bibliografía original y, 17 temas emergentes, que finalmente devinieron en 4 categorías teóricas que permiten describir la esencia, o elemento culminante del fenómeno (COLAIZZI, 1978; SANDERS, 2003). En sus narraciones acerca de su quehacer, los docentes narran acerca de sus experiencias, las cuales se enuncian bajo distintos temas emergentes. 
Tempranamente, los docentes se refieren a su "trayectoria laboral", vinculada a los años de antigüedad en el ejercicio docente, al espacio geográfico de trabajo, y al nivel educativo en donde desempeñan su tarea. Los participantes incluyen descripciones de los diferentes escenarios que van moldeando su experiencia y que ponen de manifiesto los cambios que se van sucediendo a lo largo de los años. También incluyen la reminiscencia del pasado y su inevitable comparación con los acontecimientos presentes. Por otro lado, la "capacitación docente", que se refiere a la actualización y perfeccionamiento docente, se relaciona con la necesidad de preparación específica y actualización que se considera importante como requisito para el trabajo en las aulas, y para la post-titularización. Esta necesidad de capacitación se ve teñida por el alto costo económico que poseen las capacitaciones y que genera cierta resistencia por parte del profesorado.

El tema emergente, "descripción del sí mismo", está asociado a consideraciones positivas sobre la persona y la función del profesor. Adjetivos como excelente, importante, dedicado, comprometido, exigente, responsable-, demuestran los calificativos con los que los docentes de describen a sí mismos, e incluyen sentimientos gratificantes para ellos, tales como felicidad, comodidad, tranquilidad, orgullo, así como, sentimientos negativos como el cansancio por falta de tiempo libre y estrés. Por su lado, el tema emergente "tarea docente", es mencionado en las narrativas docentes en consideración con las funciones que debe cumplir el profesorado, entre las que se mencionan guiar la construcción del saber, planificar, corregir, ayudar, contener, comprender, escuchar, conocer a los alumnos y sus necesidades, manejar a los padres, coordinar actividades con el resto de los actores de las instituciones, entre otras.

Se denomina "visión del alumno" al tema emergente que refiere a las descripciones que ofrecen los docentes de sus alumnos según diferentes características: a- según características socio-económicas, los alumnos son -humildes-; b- según sus habilidades cognitivas, tienen -alto rendimiento, debilidades en gramática y ortografía, nivel educativo bajo, carecen de vocabulario-; y c- según sus características de personalidad, los alumnos son - problemáticos, poco participativos, tienen atención dispersa, y tienen problemas con bábitos de bigiene-. En relación con el tema emergente "escuela", los docentes hacen referencia a su doble vertiente, por un lado, las condiciones edilicias de las mismas y, por otro lado, las funciones que cumple en la sociedad, incluyendo funciones - académica, de trasmisión de valores, formación de hábitos e inclusión 
social-. Otro tema de relevancia, es lo que para el profesorado supone el "ejercicio de la docencia", que se conceptualiza como una forma de vida que supone el desarrollo de la vocación, elección y profesión, cuya principal función es educar seres bumanos.

La cuestión "vincular", presente en toda la narrativa docente, se constituye en el elemento clave de su quehacer, aludiendo principalmente a la "relación docente - alumno" como vínculo fundamental para la consecución de los objetivos de aprendizaje y la adaptación al medio escolar. Esta relación entrañable, contiene una fuerte carga afectiva que impacta en el plano académico y cognitivo y moldea el proceso de aprendizaje de los estudiantes. Por otro lado, en la "relación docente - docente", el equipo de trabajo aparece ligado a la organización interna de la actividad cotidiana dentro de las escuelas, y este incluye: a- distribución de tareas, b-organización de horarios, c-intercambios de cursos y d-creación de un sistema de soporte y pilar en el quehacer docente propio y de los colegas. Por su lado, la "relación docente padres/familia", es relevante en las narraciones docentes, y considera que la visión que la familia tenga de la escuela y de los docentes, va a depender del comportamiento, la forma de aprendizaje y los vínculos que los hijos establezcan en el sistema educativo. Las narrativas también hacen alusión a la ausencia de los padres, a la situación económica de los mismos y a su falta de apoyo a la tarea docente. Finalmente, se menciona la "relación docente - directivo", que es un tipo de vínculo que actúa como facilitador u obstaculizador del trabajo docente.

Otro de los temas emergentes que atraviesa la narrativa docente está vinculado con las "condiciones laborales". Estas se asocian al contexto político que transversaliza la práctica docente. La falta de titularización, la mala remuneración económica, las dificultades existentes en cuanto a la movilidad y traslado para llegar a los lugares de trabajo, el estado de los caminos y la inseguridad son variables que se conjugan y que traen aparejados aspectos asociados al malestar docente en relación con sus funciones y tareas diarias. Por último, el tema relacionado con la "visión de la sociedad", se refiere a la percepción que tiene la sociedad sobre la tarea docente, que en general se relaciona con la desvalorización de la misma.

Al finalizar una exhaustiva descripción, estos 17 temas emergentes se redujeron a la descripción esencial y fundamental del núcleo de la experiencia, constituyéndose un modelo teórico basado en cuatro dimensiones constitutivas del ser docente y vinculadas indefectiblemente al quehacer docente: 
a- el Ser Ontológico, alude a la apropiación del individuo de sí mismo en tanto ser docente. Esta condición del ser reafirma la humanización y la vocación docente. A su vez, también pone énfasis en la principal función docente que es la de educar y formar seres humanos. El ser del docente está basado en una visión maternal de sí mismo, relacionada con valores de protección y amor.

"No quiero pasar en la vida por pasar, quiero dejar huellas en cada paso que doy, sobre todo para poder formar e incluso cambiar corazones, mentalidades, vidas, porque ser educador es tener un espíritu de servicio como ningún otro" (narrativa docente).

b- El Ser Ético, está vinculado con dar respuesta a la demanda del otro. A su vez, plantea al docente una exigencia ineludible: tratar de ser cada día más consciente de cuáles son los factores que operan sobre sí mismo y de qué manera influyen en su trabajo. El apropiarse del Ser docente se conjuga con las normas de actuación en función del deber ser, en tanto ideal del ser. La excelencia, la responsabilidad y la dedicación son los elementos que definen la función normativa del docente. La dimensión del Ser Ético está asociada a la visión altruista como elemento constitutivo del quehacer docente, en tanto tendencia a procurar el bien de los demás de una manera desinteresada.

"Estos niños luego de la escuela salían a vender o pedir en los semáforos para poder comer o bien darles el dinero a sus padres. Debíamos tratar que todas las actividades las realicen en las aulas, ya que era el único lugar que le permitía viajar, soñar, conocer otros sitios, es decir tener esperanzas. Tenía que hacer lo imposible para que esos pequeños tengan ganas de continuar en la escuela, lo cual era muy difícil" (narrativa docente).

El trabajo no regido bajo este concepto es considerado en las antípodas del quehacer docente e implica una actitud egoísta, por tanto, negativa.

"[...] observo que hay muchos colegas que no se interesan en su trabajo y esto ha perjudicado enormemente a la educación de nuestros niños. No existe una supervisión adecuada para evitar estas prácticas y lamentablemente esto va entorpeciendo el proceso de aquellos que han puesto todo su empeño" (narrativa docente) 
C- El Ser Pragmático, dimensión en la cual se enmarca y condiciona la tarea, ofrece un marco para la creación del desempeño de la actividad docente en su trayectoria, es decir, en las distintas etapas por las que el docente transcurre una vez inserto en su actividad laboral. Esta dimensión está atravesada por la reconfiguración vincular, marco de las dinámicas escolares y sociales emergentes. La tarea propia del docente - la de enseñar - aparece asociada a otras actividades que requieren del trabajo de contención social - ayudar, escuchar, atender- a los sujetos con derecho a la educación. Esta dimensión se manifiesta desde tres planos: $a$ - el plano institucional, b. el plano intervincular y c- el plano didáctico - pedagógico.

En este sentido, la configuración del ser docente tiene un encuadre institucional. El ámbito institucional, se pone de manifiesto en los tres niveles de análisis: a- como organización, b- como legalidades e historias fundantes que le dan sentido, y c- como orden de la cultura asociadas a las significaciones propias del tiempo y del espacio.

"Durante este tiempo pasé por numerosas escuelas y equipos directivos, muchos de ellos dejaron huellas profundas en mi carrera...allí estuve más de 13 años y tengo miles de anécdotas para compartirte, la que me marcó como docente" (narrativa docente)

El plano intervincular, supone una diversa y compleja trama de relaciones vinculares, entre las que, la relación con los alumnos es el vínculo fundamental. La vinculación docentealumno, se constituye como tal en tanto se establece un entramado afectivo entre ambos que es valorado positivamente por el docente. Alrededor de la misma se establece otra relación necesaria con los padres de familia. El vínculo de trabajo, por otro lado, se construye además con los pares entendidos como 'equipo de trabajo' - Ellos son los que actúan como soporte y referencia según la organización y el intercambio de tareas. También, como parte de este entramado, los directivos constituyen un eslabón fundamental en la cadena de vinculaciones. Las autoridades son quienes, claramente, pueden actuar como facilitadores u obstaculizadores de la tarea docente.

En referencia con la dimensión didáctica pedagógica, el quehacer está vinculado a una multiplicidad de tareas, tendientes a la resolución de problemas, cumpliendo funciones tales como: guía para la construcción del saber, planificador de actividades y organizador de las tareas institucionales. Asimismo, moldea las normas institucionales; maneja a los padres, 
coordina con los demás actores de la institución y brinda oportunidades. La contracara de esta función excesiva se ve reflejada en el exceso de tarea que, conjuntamente con la situación económica, produce sentimientos negativos, exacerbando el cansancio y el estrés aparejado a la falta de tiempo libre.

"[...] comienzo a elaborar la planificación de los nuevos contenidos, utilizo el libro de trabajo con los alumnos también otros, una vez que tengo todo, comienzo a armar mi carpeta didáctica, con fotocopias de actividades y con los conceptos que enseñaré pensando en una actividad importante para un día de la semana para desarrollar el tema." (narrativa docente)

d- el Ser Político, por último, es el contexto que determina, moldea y atraviesa la práctica docente. Incluye aquello sobre lo cual los profesores sienten que no tienen injerencia directa ya que supone una cierta externalidad, y la toma de decisiones que no dependen de sí mismo. En los últimos tiempos, el profesorado se ha visto inmerso en nuevas funciones tales como la alimentación, la contención social, la prevención de la salud, la lucha contra las adicciones, entre otras, dentro de su trabajo diario con los alumnos. Como consecuencia, los docentes sienten que existe un proceso de desprofesionalización de su tarea cuando se ven obligados, bajo diversas circunstancias locales, a asumir nuevos roles y trabajos para los cuales no fueron educados. De esta manera, ellos se convierten en asistentes sociales diletantes y no calificados. En este contexto, los docentes también comprenden que su alumno es un emergente del debilitamiento del lazo social y de la situación socio-económica.

En esta dimensión, los docentes comprenden que existen áreas de su trabajo profesional que están en juego. Entre ellas se destacan las relaciones de empleo, entre las que podemos mencionar las formas de contratación, remuneración, desarrollo de la carrera y estabilidad laboral. El docente como sujeto político encuentra que su labor está ajustada a las políticas gubernamentales que van configurando su ser pragmático.

"[...] somos muy mal valorados por parte de la sociedad... y nuestro estatus está por debajo" (narrativa docente).

En la tabla 1 se presentan las categorías teóricas desarrolladas desde el análisis de los temas emergentes y los agrupamientos temáticos. Aquí se puede apreciar como las cuatro dimensiones del ser docente se conjugan y funden. 
Tabla 1 - Categorías teóricas desarrolladas a partir del análisis de los temas emergentes y los agrupamientos temático

\begin{tabular}{|c|c|}
\hline \multicolumn{2}{|c|}{ Dimensión 1: Ser Ontológico } \\
\hline Docencia & $\begin{array}{l}\text { conceptualización de la docencia como forma de vida; docencia como vocación/ } \\
\text { elección/profesión; amor a la docencia; valoración positiva de la docencia; descripción de } \\
\text { la docencia como un quehacer; descripción de la aptitud para la docencia; la docencia } \\
\text { como una función maternal relacionada con los valores de amor y protección. }\end{array}$ \\
\hline \multicolumn{2}{|c|}{ Dimensión 2: Ser Ético } \\
\hline Docente & $\begin{array}{l}\text { Vision altruísta: Ilusion. y desejo; Vision contra altruísta; sentimentos; descripción de sí } \\
\text { mismo; exceso de tarea. }\end{array}$ \\
\hline Alumnos & $\begin{array}{l}\text { Vision idealizada del alumno; formas o recursos de y para aprender; descripción de los } \\
\text { alumnos, en cuanto a variables económicas, académicas; familiares; conductuales; Vision } \\
\text { del alumno como emergente del debilitamiento del lazo social y de las estructuras sociales; } \\
\text { descripción grupal de quien tienen a cargo. }\end{array}$ \\
\hline Escuela & escuela como espacio físico; función de la escuela; ambiente laboral \\
\hline \multicolumn{2}{|c|}{ Dimensión 3: Ser pragmático } \\
\hline $\begin{array}{l}\text { Trayectoria } \\
\text { laboral }\end{array}$ & $\begin{array}{l}\text { años de antigüedad; ámbito geográfico de trabajo; nivel educativo donde se desempeña; } \\
\text { transcurso por diferentes escenarios; reminiscencia del pasado diferente del presente. }\end{array}$ \\
\hline $\begin{array}{l}\text { Capacitación } \\
\text { docente }\end{array}$ & $\begin{array}{l}\text { perfeccionamiento y la actualización docente; falta de preparación especial; titularización; } \\
\text { el costo económico en relación con el nivel alcanzado. }\end{array}$ \\
\hline $\begin{array}{l}\text { Relación alumno } \\
\text { docente, equipo de } \\
\text { trabajo, directivos. }\end{array}$ & $\begin{array}{l}\text { relevancia del vínculo para la consecución de los objetivos de aprendizaje y la adaptación } \\
\text { al medio escolar, vínculo que implica una relación afectiva que impacta en el plano } \\
\text { académico y cognitivo. la organización interna del trabajo; con el equipo de trabajo se } \\
\text { construyen lazos solidarios, de soporte y referencia. }\end{array}$ \\
\hline Tarea docente & $\begin{array}{l}\text { Pragmático; ilusión, desejo y altruismo; satisfacción por la tarea realizada y los } \\
\text { sentimentos que genera; aceptación por parte de los alumnos y de los otros; la tarea } \\
\text { docente se diferencia del resto de los oficios; funciones implícitas de la tarea docente; } \\
\text { tareas extras; crisis externas que afectan la labor del docente; valoración positiva de la } \\
\text { tarea docente. }\end{array}$ \\
\hline \multicolumn{2}{|c|}{ Dimensión 4: Ser político } \\
\hline Sociedad & desvalorización social \\
\hline Educación & función esencial, abstracta e indispensable; cimiento del progreso de una sociedad. \\
\hline $\begin{array}{l}\text { Condiciones } \\
\text { laborales }\end{array}$ & bajo salario; falta de titularización \\
\hline Contexto escolar & $\begin{array}{l}\text { dificultades existentes en cuanto a traslado; estado de los caminos y posibilidad de acceso } \\
\text { por las inundaciones, transporte e inseguridad; } \\
\text { reproduce lo que a nivel social económico y cultural existe (diversidad, diferenciación } \\
\text { social, brecha entre lo público y lo privado }\end{array}$ \\
\hline
\end{tabular}

Fuente: Elaboración propia. 
Figura 1 - El ser docente analizado desde las capas concéntricas de las dimensiones del ser docente

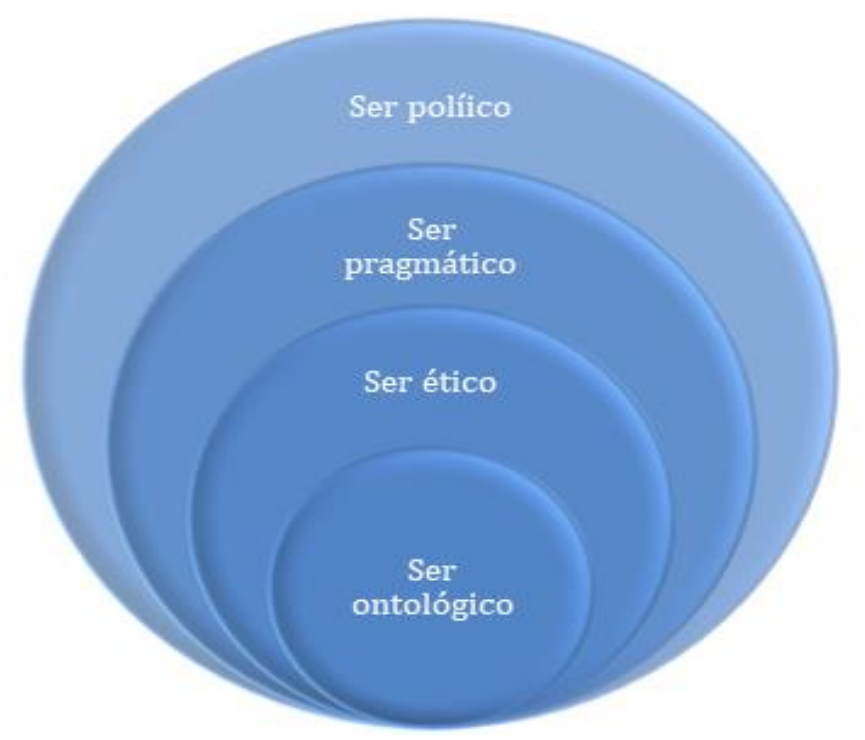

Fuente: Elaboración propia.

La figura 1 representa gráficamente las dimensiones del Ser docente.

\section{Consideraciones finales}

Los resultados obtenidos ponen en evidencia que en la narrativa acerca del quehacer docente, el profesorado y su tarea trascienden el ámbito de la clase (SAUCEDO; ALCALÁ; FERREYRA, 2019). La descripción precedente desarrolla un entramado de dimensiones y relaciones que conjugan y traspasan el hacer docente en sí mismo, pero que a la vez, lo constituyen y lo atraviesan.

El trabajo docente involucra un alto grado de subjetividad (HIPÓLITO; FERREYRA, 2010) que se constituye en una encrucijada entre el Ser Ontológico, el Ser Ético, el Ser Pragmático y el Ser Político. En esta realidad, se presenta la necesidad de construir una identidad docente diferente validada en la construcción colectiva.

La estructura del ser docente que delimita y configura el quehacer, está formada por cuatro capas concéntricas de diferente dinámica que en conjunto constituyen la identidad docente construida colectivamente desde la experiencia vivida. La dimensión política está condicionada por las características del modelo económico y el rol del Estado en relación 
con las políticas sociales y específicamente con las políticas educativas. Como señala Gil Anton (2016), los docentes construyen el perfil laboral que da sentido a su quehacer diario, a partir de la interacción con la imagen social vigente de su profesión en un momento histórico determinado (CARRERAS et al., 2017).

La dimensión del Ser Político es la que está más sujeta a los cambios producidos por el contexto social inmediato. Las coyunturas políticas, económicas y sociales modifican esta estructura constituyente del ser docente. La propia dinámica de esta configuración repercute directamente en la dimensión del Ser Pragmático en los tres planos - institucional, vincular y pedagógico - didáctico -. Lo que se pauta externamente repercute en el quehacer cotidiano de la vida escolar.

La dimensión del Ser Ético, conjuntamente con el Ser Ontológico son las dimensiones que mayor estabilidad poseen, presentan mayor resistencia a los cambios y se consolidan como refuerzos identitarios defensivos frente a las contingencias y movimientos que las otras dos dimensiones producen (CARRERAS et al., 2017). El análisis de la narrativa evidencia que los docentes constituyen un colectivo que pone de manifiesto una particular manera de resistir a los embates desde el exterior. Esta resiliencia se desarrolla continuamente frente a las lógicas y las modalidades educativas imperantes, en los casos de emergencia en la adecuación y adaptación al proyecto educativo, desde su configuración en el complejo entramado de hilos que lo atraviesan y lo definen como tal. Consecuentemente, nos hacemos eco de la definición de Bellei (2001), quien refiere que los docentes desarrollan un sociogenético instinto de conservación, alojado en la base identitaria de su Ser docente en una visión ontológica - romántica del mismo.

Las vicisitudes por la que transcurre la profesión docente, convocan a quienes se interesan por el campo educativo a la delimitación de herramientas conceptuales que abarquen las dimensiones que definen el Ser docente. Este Ser docente se configura en un marco histórico específico, así como geográfico y sociológico. Los docentes se posicionan en la resistencia romántica desde la dinámica de las subjetividades puestas en juego en su conformación identitaria, por lo que el Ser Ontológico y el Ser Ético se configuran como agentes y como cimientos de la supervivencia del docente y su tarea. 
El fortalecimiento de estas dimensiones identitarias coadyuva a la toma de conciencia como posibilitadora de la transformación social.

\section{Referencias}

ACHILLI, E. L. Investigación y formación docente. Argentina: Labor, 2008.

AGUAYO ROUSEL, H. B. Personal formador de docentes. Representaciones sociales de su profesión. Revista Electrónica Educare. México, v.24, n.1, p.1 - 18, 2020.

AGUIRRE, J., APARICIO, M.; MARSOLLIER, R. Docencia educación y escuela en riesgo: un estudio en docentes de nivel medio de Mendoza. Congreso Iberoamericano de Educación. Buenos Aires: OEI, 2010.

BARCO, S. La corriente crítica en didáctica: una mirada elíptica a la corriente técnica. In: CAMILLONI, Alicia W. et al. (Eds.). Corrientes didácticas contemporáneas. Buenos Aires: Paidós. 1996. p. $157-167$.

BELLEI, C.; VALENZUELA, J.P. ¿Están las condiciones para que la docencia sea una profesión de alto estatus en Chile? In: MARTINIC, S.; ELACQUA, G. (Ed.). ¿Fin de ciclo? Cambio en la gobernanza del sistema educativo. Santiago: UNESCO/Pontificia Universidad Católica de Chile, 2010. p. 257-284.

BELLÓ, P.; TORRES STÖCKL, C.; CARRERAS, M. P. El quehacer docente: intercambio de narrativas entre docentes latinoamericanos. (F. d. UNT, Ed.) Tucumán, Argentina: IV Congreso de Psicología del Tucumán, 2019.

CARRERAS M. P. et al. Prototipo representacional del trabajo docente: una investigación en el ámbito local. Memorias del V Congreso internacional de Psicología del Tucumán "Desafíos actuales en la Psicología. Nuevas demandas y contextos de intervención". San Miguel de Tucumán- Argentina, p. 1-3. 2017.

COLAIZZI. P.F. Psychological research as the phenomenologist views it. In: VALLE, R.S.; KING, M. (Eds), Existential-phenomenological alternatives for psychology. New York: Oxford University Press, 1978. p. 48-71.

CORRALES, J. Presidents ruling parties and party rules: a theory on the politics of economic reform in Latin America. Mimeo, 1994.

EDELSTEIN, G. E. El análisis didáctico de las prácticas de enseñanza. una referencia disciplinar para la reflexión crítica sobre el trabajo docente. Revista del Instituto de Investigaciones en ciencias de la educación. Año IX, n.17, 2000.

HEIDEGGER, M. Being and time: A translation of Sein und Zeit. SUNY Press, 1996. 
HINCHMAN, L. P.; HINCHMAN, S. K. (Eds.). SUNY series in the philosophy of the social sciences. Memory, identity, community: the idea of narrative in the human sciences. New York: State University of New York Press, 1997

MEZA RUEDA, J. L. Pedagogía Narrativa. Aproximaciones a su epistemología, su método y su uso en la escuela. Actualidades pedagógicas, n.54, p.97-105, 2009.

MOUSTAKAS, C. E. Phenomenological research methods. Thousand Oaks, Calif.: Sage, 1994

NAVARRO, J. C. Dos clases de políticas educativas: la política de las políticas públicas. In: La política de las políticas públicas: progreso económico y social en América Latina. Banco Interamericano de desarrollo. Chile: San Marino, 2006.

NOVAES, A. O. Subjetividad, representaciones sociales y posibilidades de transformación social". (Conferencia). IV Congreso internacional de Psicología del Tucumán "Cultura y subjetividad: Psicología interpelada”. San Miguel de Tucumán, Argentina, 2015.

PRIETO QUEZADA, J. C.; CARRILLO NAVARRO. Formación docente, subjetividad e incertidumbre en México. Revista Educaşão e Cultura Contemporánea, Rio de Janeiro, v.15, n.41, p.15-30, 2018.

SANDERS, C. Application of Colaizzi's method: interpretation of an auditable decision trail by a novice researcher. Contemporary nurse: A journal for the Australian nursing profession, v.14, n.3, p.292-302. doi:10.5172/conu.14.3.292. 2003

SAUCEDO, M. G.; ALACALÁ, M. T.; FERREYRA, H. A. La práctica docente que trasciende al ámbito de la clase. Las posiciones que construyen los profesores en torno a las actividades que realizan en la escuela secundaria. Revista del Instituto de Investigaciones en Educación, v. 8, n. 11, p. 49-62, 2019.

SOUSA, C. P.; VILLAS BÔAS, L. A teoria das representações sociais e o estudo do trabalho docente: os desafios de uma pesquisa em rede". Diálogo Educacional, Curitiba, v.11, n.33, p. 271-286. 2011a.

SOUSA, C. P.; VILLAS BÔAS, L.; NOVAES, A. O. Contribuções dos estudos de representações sociais para compreensão do trabalho docente. In: ALMEIDA, A. M. de O.; SANTOS, M. F. de S.; TRINDADE, Z. A. Teoria das Representações sociais: 50 anos. Brasilia: Tecknopolitik, 2011b. p. 625-644.

VAN MANEN, M. Researching lived experience: Human science for an action sensitive pedagogy. Albany, N.Y.: State University of New York Press, 1990.

RECEBIDO: 02/06/2020

APROVADO: 28/07/2020

RECEIVED: 06/02/2020

APPROVED: 07/28/2020

RECIBIDO: 02/06/2020

APROBADO: 28/07/2020 